Intersections

Canadian Journal of Music

Revue canadienne de musique
Intersections

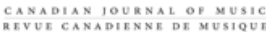

\title{
Musiques et identités remixées
}

\section{Gérald Côté}

Volume 35, numéro 1, 2015

URI : https://id.erudit.org/iderudit/1038944ar

DOI : https://doi.org/10.7202/1038944ar

Aller au sommaire du numéro

\section{Éditeur(s)}

Canadian University Music Society / Société de musique des universités canadiennes

ISSN

1911-0146 (imprimé)

1918-512X (numérique)

Découvrir la revue

Citer cet article

Côté, G. (2015). Musiques et identités remixées. Intersections, 35(1), 53-78. https://doi.org/10.7202/1038944ar

\section{Résumé de l'article}

Cet article présente une analyse contextuelle du travail créatif de quatre musiciens qui avaient comme mandat de remixer des chansons québécoises enregistrées avant les années 1960. Par le biais d'une enquête dont l'approche s'inspire de méthodes ethnographiques, l'auteur tente d'entrer dans la tête et le coeur des musiciens en vue d'identifier ce qui motive leur créativité et ce qui mène ces derniers à s'incliner vers certains modèles esthétiques plutôt que d'autres. Dans un espace social qualifié de postmoderne ou encore d'hypermoderne selon les sociologues, les motivations nous apparaissent diversifiées, fragmentées, fluctuantes et très contrastées d'un musicien à l'autre, et ce, à l'image d'une époque, d'une génération ou d'une réalité anthropologique urbaine et contemporaine. L'auteur conçoit la musique comme un phénomène multifactoriel où le social et le musical sont interdépendants, en mouvements ainsi qu'en constantes mutations.
Copyright (C Canadian University Music Society / Société de musique des universités canadiennes, 2016
Ce document est protégé par la loi sur le droit d'auteur. L'utilisation des services d'Érudit (y compris la reproduction) est assujettie à sa politique d'utilisation que vous pouvez consulter en ligne.

https://apropos.erudit.org/fr/usagers/politique-dutilisation/ 


\title{
MUSIQUES ET IDENTITÉS REMIXÉES
}

\author{
Gérald Côté
}

\section{INTRODUCTION $^{1}$}

Le projet « Remixer la chanson québécoise », une idée du musicologue Serge Lacasse, vise un triple objectif: la réalisation d'un album de remixes de chansons québécoises enregistrées avant 1960, afin de contribuer à la (re)valorisation du répertoire chansonnier québécois, et mieux comprendre les motivations au cœur du processus de création des remixeurs contemporains ${ }^{2}$.

C'est ce troisième objectif qui fait l'objet de cet article. Pour l'atteindre, nous avons mené une étude ethnographique du processus créatif des participants au projet, dont cet article rend compte. Essentiellement, nous avons demandé à 13 musiciens de créer des remixes (15) en se basant sur un ou plusieurs enregistrements de musique québécoise d'avant les années 1960. Ils étaient totalement libres d'adopter l'approche esthétique de leur choix. Les remixeurs n'avaient qu'une seule contrainte à respecter: utiliser, d'une manière ou d'une autre, des enregistrements de chansons québécoises d'avant 1960. Pour réaliser leurs remixes, ils ont généralement eu recours à leur propre équipement, mais avaient aussi accès, au besoin, aux ressources (matérielles et humaines) du Laboratoire audionumérique de recherche et de création de l'Université Laval (LARC), en particulier pour les étapes de la prise de son, du mixage et du matriçage. Épaulés par une équipe de musicologues, les remixeurs ont également pu participer à des séances d'information sur le contexte historique dans lequel ont été conçues les chansons originales, sur leurs acteurs et sur les ressources disponibles pour accéder aux enregistrements. Ils ont aussi participé de façon active à des «réunions de production» au cours desquelles plusieurs décisions ont été prises. Lors de ces réunions, les remixeurs ont pu faire entendre aux membres de l'équipe présents les diverses versions de leurs remixes au fil de leur réalisation afin de se nourrir de leurs commentaires. Finalement, une page Facebook a permis à tous de s'exprimer au cours du processus de réalisation du projet et d'avoir accès à des informations de la part des superviseurs du projet ou d'autres remixeurs. Avant de présenter les résultats de ce travail ethnographique, il convient de résumer les assises théoriques sur lesquelles repose cette étude.

1 La recherche ayant mené à la rédaction de cet article a bénéficié du soutien du Fonds de recherche sur la société et la culture (FRQ-SC).

2 Une description synthétique du projet est proposée sur le site du FRQ-SC: http://www.frqsc.gouv.qc.ca/fr/la-recherche/la-recherche-en-vedette/histoire/remixer-la-chanson-quebecoise -lihagbsj1424704005894. 


\section{IDENTITÉS, IDÉOLOGIES ET HYPERMODERNITÉ}

Le travail créatif en art s'inscrit avant tout dans une démarche identitaire soutenue. Toute démarche artistique est indissociable d'une identité profonde, laquelle se manifeste par une mise en scène particulière relative à un contexte. Aucun choix ne peut relever du vide, car s'il y a un choix, il y a nécessairement une modalité quelconque qui s'accompagne d'un discours circonstanciel.

C'est par identification directe avec des modèles en particulier (sonores en ce qui concerne notre étude) que l'individu balise certains aspects de son identité complexe: « un processus d'identification par lequel l'individu se rend semblable aux autres, assimile leurs caractéristiques, se trouve des modèles pour construire sa personnalité et se sent solidaire de certaines communautés.» (Edmond 2005, p. 3-4). Or l'environnement postmoderne accentue le phénomène d'identité multiple. À l'évidence, avec le foisonnement des réseaux et des organisations de tout acabit qui s'entrecroisent, la société contemporaine se complexifie tant à l'égard des rôles sociaux (et nous ajoutons par voie de conséquence ici), qu'à l'égard des objets culturels et de leurs référents symboliques:

D’où proviennent les «moi» complexes. Stryker (1980) suggère que le moi complexe relève d'une société complexe. Comme la société tend à se différencier en termes de groupes, d'organisations, et de rôles possibles pour les individus, ces derniers qui construisent leur «moi» à partir de ces différentes expériences deviennent eux-mêmes complexes. [...] Bien plus, à cause des réseaux de l'ère prémoderne qui s'intègrent les uns aux autres, le partage des jeux de significations ainsi que les attentes sont décuplés dans un tel contexte. (Burke et Stets 2009, p. 132)

Dans le contexte qui nous occupe, le "moi complexe» s'alimente de fragments de cultures qui ont été construits et stigmatisés par les générations posthippies, ces dernières étant inondées par les lois du marché et de la consommation. Ainsi, des masses d'identités-objets instrumentalisées sont façonnées dans ce moule environnemental et circulent allègrement par l'intermédiaire des médias qui les diffusent au profit d'une élite appelée PMC33. Comme le mentionne Timothy D. Taylor dans Music and Musical Practice in Postmodernity (2002), tout cela va concrétiser la postmodernité:

Ce sont les artistes et musiciens de la classe moyenne bénéficiant d'un important bagage culturel qui utilisent plus librement les multiples référents identitaires. Ils manipulent les identités, les sons et les instruments comme des jouets à partir desquels ils contribuent à la construction de formes esthétiques qui ont été bien caractérisées dans la vision de Jameson et d'autres réflexions postmodernes. (Taylor 2002, p. 101).

Pas étonnant que nous puissions observer une perspective relativiste chez plusieurs musiciens contemporains postmodernes. Cela se présente sous différentes facettes, entre autres, par le fait que ces derniers peuvent s'identifier à des styles ou à des référents sonores qui s'opposent dramatiquement entre

3 En référence à la génération des yuppies, «professional-managerial class». (Ehrenreich 1979). 
eux sur le plan esthétique. Conséquemment, ce genre de ballotement pourrait être perçu comme provenant de personnalités instables, complexes, porteuses de contradictions, un phénomène favorisé par l'environnement hypermoderne ${ }^{4}$, comme l'illustrent ces propos d'Aubert dans son article sur l'individu hypermoderne:

Le portrait de l'individu contemporain qui s'est ainsi dégagé présente des facettes contradictoires: centré sur la satisfaction immédiate de ses désirs et intolérant à la frustration, il poursuit cependant, dans de nouvelles formes de dépassement de soi, une quête d'Absolu, toujours d'actualité. (Aubert 2006, p 2-3)

Or, parlant du même phénomène, Lipovetsky y voit plutôt une grande mobilité, une ouverture d'esprit ainsi qu'une tolérance accrue:

Les individus sont certes plus mobiles et plus fluctuants aujourd'hui quant à leurs opinions, est-ce un mal pour autant? Sous le règne de la mode totale, l'esprit est moins ferme, mais plus réceptif à la critique, moins stable, mais plus tolérant, moins sûr de lui-même, mais plus ouvert à la différence. (Lipovetsky 2004, p. 31)

Sur le plan de l'identification à des référents musicaux stylistiques, nous remarquons que le phénomène est similaire à ce que l'on peut observer dans le domaine de la sémantique discursive. Ainsi, nous sommes en accord avec l'idée de Longhi (2008, p. 156), qui, parlant des formes sémantiques dont le "sens est en relation avec notre expérience», fait remarquer que les constructions identitaires se nourrissent d'objets possédant un potentiel discursif. Dans son essai sur la sémantique discursive (2008), ce dernier propose la notion "d'objet discursif», un concept qui, à notre avis, se transpose parfaitement aux référents stylistiques musicaux, car les objets culturels, qu'ils soient sonores ou non, constituent un moyen, voire un procédé qui s'appuie sur une forme de doxa ${ }^{5}$ implicite dans l'objet lui-même.

Dans une telle perspective, les nouvelles technologies favorisent la réconciliation symbolique des identités morcelées qui, en plus de se multiplier, prennent des formes de plus en plus excessives et extravagantes, dans un bal d'hyperactivité et d'excès collectif. Il s'agit là d'un phénomène indissociable des comportements sociaux qui s'hypertrophient au rythme d'une société d'hyperconsommation, comme le souligne Aubert:

4 L'idée de Lipovetsky autour de l'hypermodernité réside essentiellement dans une observation phénoménologique sur les comportements sociaux qui valorisent les expériences individuelles et sociales extrêmes. "L'escalade paroxystique du "toujours plus" s'est immiscée dans toutes les sphères de l'ensemble collectif” (Lipovetsky 2004, p. 53). Dans le contexte de cette recherche, certains remixeurs manipulent ces jeux interstylistiques avec plus de liberté que ne pouvaient le faire les hippies avec les référents musicaux de la fin des années 1960. En effet, ces derniers ne pouvaient pas s'identifier à fois à Janis Joplin et à Frank Sinatra.

5 Au sein des mécanismes de construction du sens en discours, la doxa est relative à une région du sens commun. 
Quand on essaie de capter ce qui caractérise le comportement de l'individu hypermoderne, produit de notre société d'hyperconsommation, sans frontières et sans limites, un des aspects qui nous a le plus frappés, c'est la notion d'excès. L'individu hypermoderne ne se contente pas de vivre dans un changement permanent, il est pris dans des situations ou développe des comportements marqués par l'excès: excès de consommation, excès de jouissance, caractérisé par un "toujours plus», une sorte de devoir de jouissance, mais aussi excès de pressions, de sollicitations, de stress. C'est un individu qui, en quête de performances toujours plus grandes, se brûle dans l'hyperactivité. (Aubert 2006, p. 4)

Dans l'esprit de Bourdieu et de Csikszentmihalyi, nous ajoutons à cela que le "moi sonore» se crée souvent entre des axes opposés, entre le rejet et l'acceptation de modèles, de référents, de situations, et ce, au sein d'une trame sociale mettant en scène des jeux de pouvoir où le matériau esthétique sert de symbole à l'affirmation d'une position personnelle ou d'un état d'esprit à l'égard d'un environnement en particulier: "Les goûts sont inséparables des dégoûts: l'aversion pour les styles de vie différents est sans doute une des plus fortes barrières entre les classes[...]. Les goûts commandent de séparer.» (Bourdieu 1984, p. 157) Pour Csikszentmihalyi (1988, p. 330) : "The work becomes part of the canon and will filter through, as one item of information in the domain, so that following generations of artists can be inspired to imitate it or reject it.»

Pour terminer ce survol sur les phénomènes de constructions identitaires, il est important de souligner qu'il existe un aspect idéologique prépondérant aux métissages musicaux ${ }^{6}$. Comme nous l'avons déjà évoqué, nous croyons qu'une fois réunis, les éléments constituant un nouvel assemblage sonore deviennent significatifs sur le plan symbolique. Par exemple, dans un contexte socioéconomique particulier, un métissage peut s'avérer le modèle d'un monde idéalisé, comme pour Dizzy Gillespie, dont le métissage entre la musique cubaine et le jazz new-yorkais converge avec son discours idéologique de circonstance sur l'unité:

Mesdames et Messieurs, nous sommes ici ce soir pour démontrer qu'en tant que frères, un échange est possible s'il existe un esprit d'unité. Si nos gouvernements respectifs ne peuvent se donner la main dans un esprit fraternel, nous allons leur montrer comment le faire, ce soir. (Gillespie, dans Polanetska 1988)

En fait, toute l'histoire des musiques afro-américaines témoigne d'une dynamique d'opposition et de réconciliation entre le Noir et le Blanc. En effet, nous pouvons observer dans le contexte de l'esclavagisme et de la ségrégation que les différents métissages entre la musique européenne et celle des Africains-Américains renferment des discours idéologiques particuliers, et ce, tant pour les Africains-Américains qui intègrent des éléments de la culture européenne que pour les musiciens américains blancs qui introduisent des éléments afro-américains dans leur musique. Dans Jazz vu de l'intérieur, Côté (2011, p. 8) illustre 2003). 
ce phénomène identitaire relatif à ces deux cultures qui ont fusionné leur musique aux États-Unis.

Par conséquent, à l'ensemble de ces réflexions sur les idéologies et le métissage, ainsi que sur la postmodernité et l'hypermodernité en relation avec le phénomène de construction identitaire, nous croyons que, parce qu'ils vont mélanger des référents sonores très diversifiés, les remixeurs, pour ce projet, vont inévitablement refléter une société complexe, donnant lieu à des «moi» construits autour d'objets-référents multiples, devenant eux-mêmes des entités remixées complexes. Il faut ajouter à cela que le pouvoir qu'offrent les nouvelles technologies va grandement leur faciliter la tâche quant à cet ultime objectif autour du processus créatif qui est celui de réconcilier le monde, leur monde.

\section{Note additionnelle sur l'approche}

Nous croyons qu'il ne peut exister aucune forme d'étanchéité entre le processus créatif, sa réception et son contexte. Certes, il peut y avoir toute une gamme de possibilités d'interprétation relative à un phénomène sonore, mais cela ne signifie pas pour autant que nous pouvons séparer les différents axes du processus créatif sans le dénaturer. Il est aussi impossible de dissocier l'objet de celui qui tente de l'analyser. Par conséquent nous nous sommes inspirés du modèle de Csikszentmihalyi (1988, p. 329) que nous avons adapté tout en y ajoutant certains éléments susceptibles d'illustrer ce qui nous apparaît être essentiel aux

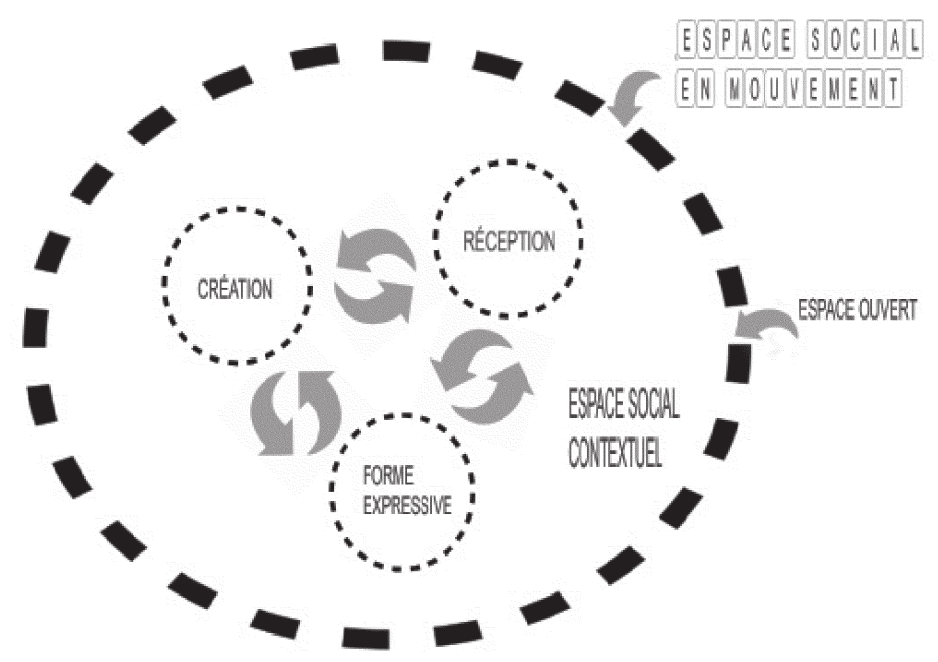

Figure 1: Modèle du processus créatif (adapté de Csikszentmihalyi)

phénomènes relevant du processus créatif relatif à notre enquête (Figure 1).

Dans ce schéma, tous les éléments sont des espaces ouverts et «interpénétrables» (comme le suggèrent les pointillés). Le tout est disposé dans un espace ouvert correspondant à un contexte social en continuelle transformation («Espace social en mouvement»), illustrant ainsi que toute fixité relève d'une 
pure abstraction mentale chimérique, car nous croyons que les mouvements et les transformations sont intrinsèques aux phénomènes culturels. Finalement, ce que nous appelons «forme expressive» dans le tableau, est aussi présenté avec des pointillés qui ouvrent sur le grand ensemble de l'espace social en mouvement.

C'est donc dans ce contexte que nous réaliserons l'analyse du processus créatif des remixeurs-créateurs en nous focalisant essentiellement sur ces deux axes:

Axe 1) Le processus créatif vu comme un phénomène de métissage culturel ou de combinaison entre éléments stylistiques dans le contexte postmoderne et hypermoderne;

Axe 2) L'affirmation identitaire manifeste dans les nouveaux assemblages sonores.

En résumé, notre approche conçoit le processus créatif au sein d'un espace où tous ses mécanismes sont en situation d'interpénétration et de mouvement, situation complexe pour laquelle le chercheur assume un angle d'observation en particulier - non neutre - c'est-à-dire que, pour cet exercice, le chercheur a choisi de mettre en relief les éléments identitaires et idéologiques relatifs à la création de nouvelles formes sonores métissées, et ce, dans le contexte de la postmodernité, de l'hypermodernité et du développement exponentiel des technologies de l'information et de production.

Comme nous l'avons évoqué, c'est à partir d'une étude ethnographique que nous avons pu recueillir les données principales aux fins d'analyse et d'interprétation. Nous avons donc mis en place un large dispositif afin de colliger de l'information susceptible de nous éclairer sur certains aspects liés au processus créatif que nous venons de présenter. Ainsi, sur une période de plus de 36 mois, nous avons effectué une série d'entrevues, individuelles et semi-dirigées avec des moments non structurés ${ }^{7}$ d'une durée variant de 45 minutes à deux heures. Ces entrevues ${ }^{8}$ ont été faites par Skype et aussi en personne avec le concours d'une étudiante au doctorat de l'Université Laval, Méi-Ra St-Laurent. Aussi nous avons enregistré huit rencontres de production, lors desquelles nous utilisions la méthode d'observation participante, aussi échelonnées sur une période 36 mois et qui ont eu lieu à la Faculté de musique de l'Université Laval9. De plus, nous avons élaboré un questionnaire qualitatif à questions fixes orienté vers le travail créatif des remixeurs en regard des principales étapes du processus de création. Nous avons aussi demandé aux remixeurs de produire un journal individuel ainsi que de participer à un espace d'échange sur Facebook. Finalement, nous avons enregistré les séances de mixage au LARC avec la

7 Privilégiant « un contact personnel avec les sujets de la recherche, principalement par le biais d'entretiens et par l'observation des pratiques dans les milieux mêmes où évoluent les acteurs» (Paillé et Mucchielli 2008, p. 75).

8 Deux entrevues par remixeur, une au début du de la production et une autre une fois la production terminée. Dans certains cas, des entrevues informelles ont été ajoutées afin de compléter les informations nécessaires pour bien comprendre leur démarche créative. Cela est le cas pour Rem 2 et Rem3.

9 Ces rencontres entre les remixeurs portaient essentiellement sur des points techniques relatifs à leur projet de composition. 
participation de Serges Samson, le technicien en travaux d'enseignement et de recherche de la Faculté de musique de l'Université Laval, avec qui nous avons aussi eu des entretiens non directifs sur le travail des remixeurs. L'ensemble de la démarche ethnographique nous a fourni une masse d'information que nous avons organisée par thèmes ${ }^{10}$ dans une base de données, grâce à la collaboration du doctorant Yannick Lapointe. Concernant l'analyse et l'interprétation du contenu ainsi que des données, une étape de validation a été faite de manière informelle sur une période d'une année afin de vérifier les éléments pouvant faire l'objet d'interprétation de la part de l'auteur.

\section{QUATRE REMIXEURS, QUATRE CONCEPTS: INTRODUCTION AUX RÉSULTATS ETHNOGRAPHIQUES}

Pour cet exercice, nous avons choisi d'examiner le travail de quatre remixeurs parmi les 13 qui ont produit des pièces dans le cadre du projet. Nous croyons que cela est largement suffisant pour soutenir la perspective théorique qui nous intéresse. Pour chacun d'eux, nous allons nous attarder sur une pièce en particulier. Ces remixeurs ont été retenus pour la diversité qu'ils représentent ainsi qu'en raison du grand contraste existant entre leurs démarches artistiques respectives. Cet éclectisme conceptuel est significatif pour nous parce qu'il permet d'identifier une structure comportementale commune malgré les écarts stylistiques, esthétiques ou idéologiques. Nous ajoutons que, parce qu'ils témoignent d'un spectre étendu pouvant illustrer le phénomène des identités multiples dans un espace postmoderne, ces quatre remixeurs sont représentatifs de l'ensemble des musiciens du groupe qui partagent des cheminements créatifs comparables. Leurs origines, leur âge, leur culture, leur formation et leur profil s'impriment autant dans leurs idéaux que dans leur musique, ce qui nous permet d'établir des liens étroits entre des identités, des espaces sociaux complexes et des formes musicales métissées. Afin de respecter l'anonymat des candidats, nous les avons désignés par une abréviation ${ }^{11}$. Nous vous les présentons ici avec leur choix de pièces tout en identifiant les principaux outils qu'ils ont utilisés ainsi que l'essentiel de leurs opérations techniques en lien avec ce que nous croyons être un facteur identitaire prédominant.

Le premier (Rem1) est le plus âgé du groupe. Témoin des turbulences de la fin des années 1960 et du début des années 1970, ce remixeur travaille avec des tables tournantes analogiques et des prises de son réalisées en studio, ce qui nous permet d'observer le processus créatif en lien avec les premières techniques de remixage. L'univers de sa composition mélange une musique jazz-rock teintée de blues avec une chanson d'Eugène Daigneau sur 78 tours, plus précisément, "J'voudrais m'y marier", une pièce à caractère humoristique qui a été traitée de différentes manières grâce à des jeux de manipulation obtenus à l'aide d'une table tournante.

10 Principalement: les étapes du processus créatif, la technologie utilisée, les influences, la production et le mixage.

11 Pour «Remixeur $n^{o s}$ 1, 2, 3, 4", nous avons utilisé Rem1-Rem2-Rem3-Rem4. Ils sont tous au masculin pour faciliter la lecture. 
Pour Rem1, créer c'est «vraiment un processus aléatoire. Pour moi il s'agit de retenir des éléments qui permettent de faire une synthèse [...] des éléments pouvant bien se mélanger entre eux selon les possibilités et les contraintes de la technologie et de mes trucs que j'ai développés en jouant avec la manipulation d'une table tournante» (Rem1, Blogue des Remixeurs, 30 novembre 2011). Or c'est dans cet esprit qu'il a choisi une trentaine de "galettes originales ${ }^{12}$ » tout en s'efforçant de «s'éloigner des clichés de l'époque». Rem 1 s'inspire donc d'éléments qu'il sélectionne selon les possibilités techniques qui vont lui permettre de construire des ambiances particulières, mais il choisit aussi consciencieusement des référents stylistiques afro-américains selon leur capacité à pouvoir exprimer une critique sociohistorique, un aspect déterminant de sa démarche que nous allons développer plus tard:

Après, je les ai toutes écoutées à [la vitesse] 33 tours [...] parce que j'aime les ralentir. J'en ai trouvé une qui, une fois ralentie à 33 tours, donnait un riff de blues. C’est exactement ce que je cherchais. Depuis le début de ça, j'étais à la recherche du côté nègre blanc d'Amérique et j'ai fini par trouver une pompe ${ }^{13}$ qui, une fois ralentie à 33 tours, donnait un beat de blues. (Rencontre de groupe, 15 octobre 2012)

À cette trame, qu'il a travaillée chez lui avec son propre équipement, s'ajouteront des pistes qu'il va enregistrer au LARC avec un groupe de musiciens.

Rem2 est un musicien originaire de Cuba. Il est actuellement inscrit à un doctorat dont l'objectif est de perfectionner son langage jazz, et ce, bien qu'il s'intéresse aux musiques populaires et traditionnelles. Il connaît très peu le monde des technologies de remixage, mais l'exercice constitue un défi personnel pour lui ainsi qu'une excellente occasion de se perfectionner à cet égard grâce aux collaborations que lui offre le projet. Il a choisi la pièce "J'ai un bouton sur le bout de la langue» de Madame Bolduc, qu'il a jazzée tout en y ajoutant des éléments latins et africains. À partir de l'enregistrement original de Madame Bolduc, il a synchronisé un arrangement qui avait été préparé à l'avance par un autre remixeur du projet dans son studio maison avec le logiciel Cubase et que Rem2 a par la suite développé et enregistré dans un autre studio avec son groupe de musique cubaine.

Son travail créatif a pour point de départ une mélodie sur laquelle il pourra élaborer une construction harmonique plus sophistiquée:

Quand je suis dans un processus de création [...], je commence par jouer une mélodie afin de la travailler. Je ne dis pas que la mélodie et l'harmonie vont arriver tout d'un coup comme si c'était déjà fait. Quand je compose une chanson, c'est un processus de travail. Je commence avec les changements d'accords pour voir si cela fonctionne avec la mélodie. (Entrevue avec Gérald Côté, 5 juin 2013)

Bien que soucieux de faire un arrangement harmoniquement sophistiqué à partir de la mélodie, sa musique va aussi lui permettre de réunir deux mondes,

12 Son expression, en parlant des disques 78-tours.

13 En musique, «faire la pompe» consiste à marquer les temps avec les notes de basse. 
soit celui de ses origines avec celui de sa terre d'accueil, une expérimentation qu'il avait déjà faite antérieurement d'ailleurs:

On avait mélangé le folklore québécois avec le folklore cubain. On avait une chanson, "Au son des bongos", qui parle pas mal de l'esprit de notre groupe [...] et qui représente l'amitié entre Cubains et Québécois : ainsi, on a mélangé «Swing la baquaise dans le fond d'la boîte à bois » avec la chanson «Guantanemera». (Entrevue avec Méi-Ra St-Laurent, 18 février 2014)

Ainsi, dans la pièce produite pour le projet, on retrouve un arrangement jazz latino sur la piste mélodique nettoyée ${ }^{14}$ de la Bolduc, le tout ornementé de quelques éléments africains évoquant son héritage afro-cubain:

Premièrement, c'est de chercher l'harmonie originale. Je crois que c'est en Do. Et ensuite ce que j'aime faire, c'est partir d'une mélodie et changer l'harmonie avec des accords brisés (un accord naturel avec une basse différente), puis changer la rythmique, avec bien sûr l'héritage cubain/afrocubain. (Entrevue avec Méi-Ra St-Laurent, 18 février 2014)

Rem3 est d'origine maghrébine. C'est en 2013 qu'il termine ses certificats en réalisation audionumérique et en culture musicale à l'Université Laval dans l'objectif de pouvoir produire et réaliser sa musique. Il a lui aussi choisi une pièce de la Bolduc. C'est à partir de la chanson «Maurice Richard» qu'il a créé un arrangement qu'il a synchronisé avec la piste initiale, mais cependant avec un logiciel différent, Logic Pro d'Apple. Comme c'est le cas pour Rem2, son point de départ créatif est une mélodie, mais qu'il a choisie pour une raison différente, c'est-à-dire parce qu'elle s'adresse à tous et qu'elle est facile à mémoriser. Il s'agit pour lui d'un processus très conscient et dont l'objectif est de rejoindre le plus large public possible grâce à une formule accessible qu'il souhaite commerciale.

Moi, j'aime surtout les mélodies dont on peut rapidement se rappeler. Je n'essaie pas de compliquer les mélodies, je n'essaie pas de trouver des accords très intellectuels pour montrer que j'ai fait l'effort de faire une composition. J'essaie vraiment de faire le plus simple possible et qu'on se souvient de la mélodie. Il y a toujours une ligne mélodique qui est au cœur de la chanson et que l'on retrouve surtout dans le refrain. C'est surtout là que ça se passe vraiment [...] une mélodie qui doit être facile à apprendre, belle, commerciale et moderne. (Entrevue avec Gérald Côté, 8 juillet 2013)

Il va ensuite ajouter à son arrangement des éléments plus rythmés, qui «groovent», et ce, sur une harmonisation sans prétention:

Mais moi, pour ce projet, je voulais faire un truc qui sonne bien, qui a l'air d'une chanson je dirais un peu pop, un peu commerciale, qui sonne bien

14 À l'aide de logiciels spécialisés, la piste originale est traitée de manière à en éliminer le bruit de fond et à mettre au premier plan la voix de la Bolduc, et ce, tout en s'assurant que la musique originale n'entre pas en conflit avec le nouvel arrangement. En outre, et comme on l'a déjà évoqué, le travail de synchronisation de la trame d'origine avec l'arrangement a été réalisé en collaboration avec un autre remixeur de l'équipe. 
[...] Donc, j'ai écouté pas mal de chansons de La Bolduc et j’ai trouvé cette chanson où il y avait un rythme qui allait bien. Je veux dire, un tempo qui sera facile à insérer dans un remix. Et aussi, ça suivait un enchaînement d'accords, des accords majeurs, mais je les ai transformés (parfois) en accords mineurs, et les mineurs en accords majeurs. Mais, c'était des accords assez pop. [...] Et bon, j'ai juste pris le petit bout de la chanson de La Bolduc, et j’ai travaillé sur ça surtout. Parce que ça allait devenir le refrain. Donc [je me disais] que si je fais un très bon refrain, accrocheur, facile, c'est sûr que la chanson va être bonne [...]. Puis après, je vais ajouter d'autres éléments, mais c'était vraiment à partir du refrain que je voulais avoir un bon groove, un bon rythme. (Entrevue avec Méi-Ra St-Laurent, 28 février 2014)

Or sa production est motivée par une démarche spirituelle en lien avec le prédicateur musulman Amrokh Khalid ${ }^{15}$. En effet, par ses choix stylistiques, il cherche à contrer les imageries préjudiciables autour de l'intégrisme, ce qui converge avec la vision moderne de l'islam promue par Khalid, idée que nous allons développer davantage dans la prochaine section.

Rem4 est d'origine roumaine. Il a terminé une maîtrise sur mesure à l'Université Laval sur le travail de matriçage dans le contexte de l'effervescence des studios maison de la dernière décennie. Il a choisi la pièce "Chant d'automne» de Raymond Berthiaume, qu'il a mixée avec un arrangement qui s'inspire, entre autres, de «Mushroom Jazz» par Mark Farina (hip-hop UK 1990) et des compilations Jazz Cafe. Sa démarche artistique révèle un intérêt indéniable pour la qualité des textures, des timbres sonores et du rythme à partir desquels il s'ingénie à élaborer des ambiances cohérentes, et ce, en prenant comme modèles les référents stylistiques qu'il a soigneusement choisis. Il travaille avec le logiciel Live d'Ableton et le contrôleur AKAI APC 40, qui sont largement utilisés dans l'univers techno. Rem4 s'intéresse aux nouvelles technologies de production particulièrement pour ce qu'elles peuvent apporter sur le plan du renouvellement du modus operandi des musiciens-utilisateurs de studio maison $^{16}$. Peut-être parce qu'il a une formation en philosophie, Rem4 demeure très réfléchi quand il est question de définir l'art et le geste créatif, les deux étant pour lui indissociables:

Pour moi, il est difficile de donner une définition intelligente de l'art en général. La même chose s’applique à la création musicale. En la définissant, je risque indéniablement de lui amputer un aspect quelconque. En cherchant à lui donner une définition qui inclurait tous les aspects possibles, il me faudrait viser tellement large que le concept en deviendrait une «nondéfinition». (Correspondance entre Gérald Côté et Rem4, 3 octobre 2014)

15 Le Time Magazine (mai 2007) le classe comme un des hommes les plus influents au monde. Khalid propose une alternative à l'islam fondamentaliste où l'amour et le bien-être, fondés sur un mode de vie moderne, constitue une vision progressiste de la foi musulmane qui va rejoindre des millions de jeunes parmi les nouvelles générations de croyants.

16 Dans son mémoire de maîtrise, Rem4 travaille à une méthode de production où le matriçage s'opère simultanément avec l'étape de mixage des pistes. 
Dans une autre entrevue, il nous expose de manière plus pragmatique la façon dont il conçoit son propre processus créatif:

Je n'ai pas une conception définie ou une philosophie esthétique en particulier, référant à une forme de dadaïsme par exemple. Je mélange des éléments sonores, des référents et des modèles que j'aime et j'utilise des ambiances qui m'inspirent. Aujourd'hui j'aime les sons doux, ronds, calmants, après une période de ma vie où je m'intéressais à une musique plus dynamique, plus musclée. (Entrevue avec Gérald Côté, 4 octobre 2014)

Plus tard, il détaille davantage quelques-unes des étapes de son modus operandi qui illustre sa manière très intuitive de travailler avec les échantillons, c'està-dire sans autre stratégie que la satisfaction personnelle d'un résultat sonore qu'il construit une étape à la fois :

Mais après ça, quand j'ai senti que j'avais assez bien intégré la voix [...] j'en ai profité pour faire une intro que j'aimais vraiment. [...] Mais, j’ai quand même continué à utiliser les échantillons. [...] Pour la fin (de la composition), j'ai pris des échantillons (dans la pièce) qu'on ne peut pas reconnâ̂tre [...]. (Entrevue avec Méi-Ra St-Laurent, 8 avril 2014)

Rem4 nous confiera en entrevue que les pièces récentes mises sur Internet et qu'il a créées en émulant la musique ambiante électrolounge, la soul-pop ainsi que la dance music sont antagoniques aux styles «trash» qui pourtant le stimulaient tout récemment. Or, nous croyons que ce qui le distingue semble relever d'une conception personnelle de l'art où se manifeste une forme de fuite du sens commun. Ainsi, bien qu'il considère les sonorités marginales comme étant des éléments inspirants sur le moment par leur qualité timbrale, tout cela semble très relatif pour lui, créant de cette manière une certaine distance face à ses propres choix esthétiques, et ce, au profit d'une approche exploratoire et plus ludique de la création artistique.

\section{FrAgMENTS DE MUSIQUE, FRAGMENTS D'IDENTITÉ}

Toutes conceptions musicales sont des constructions qui se font à l'intersection de l'expérience individuelle et des discours sur l'expérience collective. (Côté 1998, p. 145)

L'absence de sens ne peut exister dans aucune forme de démarche créatrice, qu'elle soit musicale ou autre ${ }^{17}$. Nous croyons que l'art sans contenu significatif relève d'un concept illusoire, car toute manifestation artistique appartient nécessairement à un contexte corrélatif d'un passé délimité par une pensée de nature réductrice ou d'un futur imaginé, mais qui surtout contient de l'humanité, individuelle ou sociale, une pensée à retrouver, une pensée indivisible en fragments quelconques, comme nous sommes trop souvent portés à le faire.

17 Lorsqu'elle repose sur l'intention philosophique "de ne pas vouloir donner du sens », cela est déjà porteur d'une signification, c'est-à-dire celle de ne pas vouloir en créer justement, ce qui relève nécessairement d'une vision de l'art qui s'inscrit dans un contexte sociohistorique particulier. 
Voilà pourquoi nous croyons qu'il nous faut réunir, du moins du mieux que l'on peut, les éléments musicaux, contextuels, philosophiques, identitaires et idéologiques, qui une fois défragmentés, nous l'espérons, pourront contribuer à la restitution d'un sens présent dans le jeu créatif, ou du moins d'une interprétation éclairée de ce sens.

Ainsi, dans l'optique de cette recherche, le processus créatif est envisagé comme un geste de réconciliation entre les différents fragments d'identités qui traînent dans l'espace postmoderne et hypermoderne. Ces jeux de combinaison se sont faits à partir de matériaux composites qui contiennent forcément du sens pour les remixeurs. Ce sens apparaît, nous le soulignons, dans les rapports existant entre ces derniers et les référents sonores qu'ils ont eux-mêmes choisis pour façonner leur assemblage. Or, dans l'objectif de retrouver quelques aspects essentiels de ce sens, pour les prochains paragraphes et pour chacun des remixeurs, nous avons organisé en trois parties les éléments recueillis par l'ethnographie:

a) présentation et contexte

b) esthétique, idéologie et identités

c) nature du métissage

\section{Premier Remixeur : Remi}

\section{Présentation et contexte}

Remı a su trouver un référent symbolique dans la banque d'enregistrements qui reflétait bien son expérience relative aux mouvements idéologiques des années 1960-70. Ce dernier était un témoin immédiat du mouvement de contestation général de la deuxième demie des années 1960, où toute une génération avait remis en cause non seulement le système d'exploitation capitaliste, mais aussi les mœurs et la morale chrétienne au moment de l'arrivée de la pilule anticonceptionnelle et du rejet de ce pouvoir religieux d'après-guerre. Au Québec, ce rejet avait pris une dimension particulière à la suite de "la Grande Noirceur» sous le règne concerté de Duplessis et du pouvoir clérical:

Une partie du clergé se mêle des élections, appuie des candidats censément acceptables et vilipende les autres. Le libéralisme catholique étant une étiquette fort élastique on l'utilise pour dénoncer l'adversaire [...]. Mais dans la réalité profonde, le pouvoir du clergé est de plus en plus confirmé, celui des politiciens aussi, dans une solide division du travail. (Dumont 1996, p. 226)

Cette association politico-religieuse avait d'ailleurs provoqué une première vague de dissension qui s'était manifestée avec éclat dans le célèbre Refus global (Borduas 1948). Ce n'est donc pas étonnant que Rem1 ait choisi une chanson à boire, un référent à caractère hédoniste possédant à l'évidence une valeur symbolique anticléricale pour lui :

Et l'autre en fait, c'est une espèce de pièce qui est un peu étonnante. C'est Eugène Daigneault avec une chanson à boire qui se moque des curés dans une espèce de pseudo chant grégorien modal à l'orgue. Il fait comme du 
chant grégorien, mais en fait, il parle d'embrasser sa blonde, mais pas à la manière des bonnes sœurs. (Rencontre de groupe, 15 octobre 2012)

\section{Esthétique, idéologie et identités}

Héritier de l'esthétique de l'époque contestataire de la fin des années 1960, Rem 1 va s'inspirer principalement de deux styles musicaux afro-américains, des styles indissociables des mouvements contestataires de la fin des années 1960 et du début des années 1970 qui, comme on sait, ont été associés aux musiques noires devenues symboles anticapitalistes à l'époque:

Moi, en fait, je pensais prendre mon band de blues et qu'on se pratique sur cette minute et demie et qu'on le canne ${ }^{18}$ bien comme il faut, mais que tout ce mix-là soit juste dans le côté gauche. Dans le côté droit, tu n’aurais que l'espèce de ragtime. Ben, l'idée de base provient, cela a toujours été une chose qui m'obsède beaucoup dans la musique canadienne-française, de sa négritude, dans le sens noble du terme [...]. C’est son côté «black» un peu, son côté «root». (Rencontre de groupe, 15 octobre 2012)

Or ses intentions étaient manifestes dès le départ, c'est-à-dire que dans ses premières explorations, il espérait trouver une forme afro-américaine spécifique dans le répertoire canadien-français et dont le contenu pouvait s'arrimer avec sa perspective idéologique:

[...] finalement, je me suis rabattu sur l'autre pièce, qui est très anticléricale. Parce qu'une des choses dans l'histoire du Québec de cette époquelà, c'est l'espèce de mythe du petit peuple soumis sous l'écrou du curé pis tout ça. Ben en fait, j'ai dû abandonner l'espoir de trouver quelque chose de très «black» pis d'aller vers quelque chose de plus anticlérical un peu. Il y a comme eu un «shift» vers l'intention, parce que je n'ai pas trouvé tout à fait ce que je cherchais. En fait, j'ai trouvé juste un tiroir de vinyles. Peut-être j'aurais pu trouver d'autres disques, où il avait peut-être même des blues primitifs au Québec. Mais moi, on m’a dit qu'il n'y en avait pas vraiment. (Entrevue avec Méi-Ra St-Laurent, 3 avril 2014)

\section{Nature du métissage}

Dans la démarche créative de Rem1, on peut observer une motivation particulière qui se trouve directement reliée à son regard sociocritique concernant deux périodes distinctes de l'histoire du Québec, la période du comédien chanteur Eugène Daigneault (1895-1960), où ce dernier s'opposait à sa manière au pouvoir de l'époque, c'est-à-dire au moyen de l'humour et de l'ironie, et celle de la post-Révolution tranquille (1965-1970), qui avait troqué son calme pour un peu plus de turbulence idéologique dans le contexte du Flower Power. Dans la musique de Rem1, le rejet de la morale religieuse s'exprime musicalement par le mélange d'une chanson à boire provenant du disque de Daigneault et du son «bluesé» faisant écho à la période de contestation hippie. Son remix musical s'est donc construit autour de l'idée du rejet des valeurs cléricales ayant marqué le Québec et son histoire jusqu'à aujourd'hui. Tout cela concorde avec

18 Fixé par enregistrement. 
l'esprit de l'époque dite contestataire de la fin des années 1960 qui, à n'en pas douter, a nourri l'identité sonore de sa composition.

\section{DEUXIÈME REMIXEUR : REM2}

\section{Présentation et contexte}

C'est à Cuba que le deuxième remixeur a eu ses premières expériences comme musicien populaire avant de faire des études à Santiago de Cuba au Conservatorio de musica Esteban Salas. Après son immigration au Canada, il a poursuivi des études en jazz à l'Université de Sherbrooke, puis à l'Université Laval, à Québec. Pour mieux saisir sa démarche artistique, il faut considérer la conjoncture sociopolitique cubaine où l'enseignement de la musique européenne dite classique est privilégié au détriment des autres styles. Plus particulièrement, la musique populaire et le jazz sont très mal vus par les institutions officielles, et ce, pour des raisons que l'on peut facilement comprendre: dans le contexte de la Guerre froide et de concert avec l'idéologie communiste, l'État cubain cherche à se démarquer à la fois du capitalisme et, par association, de toute forme de musique américaine incluant celle des musiciens afro-américains, comme le jazz, le blues et leurs dérivés. À cause de cela, au moment où Rem2 était à Cuba, l'apprentissage des "autres musiques» se faisait dans la rue et souvent avec une attitude sociale qui s'opposait au pouvoir politique.

Lors de ses premières années au Québec, Rem2 a survécu financièrement surtout grâce à la musique populaire. En tant que codirecteur de sa thèse de doctorat, j'ai eu l'occasion de me rendre à Cuba avec lui afin d'encadrer son enquête ethnographique portant sur le pianiste de jazz Emiliano Salvador ${ }^{19}$, musicien qui avait été mis à l'écart par l'État et auquel il s'identifie fortement d'ailleurs. Sur le terrain, il m'est apparu évident que la position idéologique de Rem2 est manifeste dans l'ensemble de sa démarche artistique. Il n'est pas surprenant non plus que son point de vue politique anticastriste, partagé d'ailleurs par son entourage, soit étroitement relié à son expérience de musicien marginal à Cuba :

On essayait de faire ça en multipiste, mais ça ne marchait pas. On perdait de la qualité. On passait des nuits blanches et à la fin le produit était vraiment merdique. C'était la seule façon d'enregistrer des chansons parce qu'on n'avait pas l'appui du gouvernement pour aller dans de vrais studios. Notre musique n'était pas encadrée dans ce que voulait le gouvernement qui passait à la radio. On était marginal. (Entrevue avec Gérald Côté, 6 mai 2013)

\section{Esthétique, idéologie et identités}

Comme pour la musique de la côte ouest-africaine, la danse est indissociable de la musique pour les Cubains. Toute l'histoire de la musique de Cuba en est la parfaite démonstration: du son au mambo, jusqu'à la salsa en passant par le cha-cha-cha, la danse constitue la fonction principale, jusqu'au point

19 Enquête de terrain réalisée en mars 2014 sur le langage musical et les influences esthétiques d'Emiliano Salvador. 
même d'engendrer un véritable cliché international. Dans ce contexte, l'élément «dansant» demeure un critère important dans le choix de la pièce de Rem2. Il souhaite refléter cette fonction vitale de la musique cubaine telle qu'il la conçoit, car cela fait partie intégrante de ses préoccupations professionnelles au Québec:

J'ai une bonne réception malgré le fait que quand on entend la musique cubaine, on se met des souliers de danse. C'est vrai. Quand j'ai commencé avec mon groupe [...], je voulais faire des choses en $3 / 4$, mais les gens voulaient danser. On a changé le répertoire pour de la musique qui le permettait. C'est un processus de création qui me poussait à faire des chansons pour un public qui voulait danser sur de la vraie musique cubaine. (Entrevue avec Méi-Ra St-Laurent, 18 février 2014)

Lorsqu'il parle de son choix de la chanson de Madame Bolduc pour son remix, on constate qu'il est motivé par la dimension festive de cette musique et à laquelle d'ailleurs il n'a aucune difficulté à s'identifier:

Le folklore québécois, c'est joyeux, et chez nous, c'est la même chose. C'est festif, c'est latin. Vous êtes des Latins, nous sommes des Latins, pis il y a un point en commun: on bouge les fesses. Quand tu es motivé, ton processus de création est déterminé. Tu t'imprègnes de ce qui se passe autour de toi. (Entrevue avec Méi-Ra St-Laurent, 18 février 2014)

Un autre élément possédant une valeur identitaire et idéologique importante pour Rem 2 est l'utilisation du jazz, qui se manifeste dans sa composition surtout par la recherche harmonique relative au langage du jazz moderne, un langage qu'il a intégré pendant sa formation scolaire et son expérience de musicien à Cuba:

Ce que j'ai trouvé l'fun avec La Bolduc, c'est que c'est facile à faire des changements harmoniques, ça restait dans une tonalité normale et il y avait pas trop de changements de mesure comme cela arrive parfois dans le folklore. (Entrevue avec Méi-Ra St-Laurent, 18 février 2014)

Pour mon remix, j'étais en train d'écouter un disque de Chucho Valdez ${ }^{20}$ et j’ai retenu un élément de la batterie. Je me suis dit que ça pourrait bien se marier. J’ai pris cet élément de la batterie de Chucho et je l'ai mélangé avec la musique de Benny Moré21 qui appartient vraiment aux années cinquante. Par la suite, j’ai ajouté une base harmonique par-dessus. (Entrevue avec Gérald Côté, 6 mai 2013)

Ainsi, les référents idéologico-esthétiques de la musique de danse cubaine, du jazz et du folklore québécois sont réconciliés dans le remix de Rem2. La mise en scène sonore de sa composition relève à la fois de choix personnels qui sont antagoniques au sein de la trame sociopolitique cubaine et d'une dynamique d'intégration sociale en situation d'immigration au Québec comme nous l’avons évoqué précédemment.

20 Un des plus importants pianistes de jazz cubain contemporain.

21 Surnommé le "barbare du mambo", il est considéré comme l'un des plus importants chanteurs de musiques populaires cubaines. 
Il faut ajouter, pour compléter, que la création de Rem2 intègre un élément stylistique relevant directement des phénomènes postmodernes de métissage en lien avec la globalisation des marchés. Concrètement, l'auteur a saisi l'occasion esthétique que permettait son cadre mélodico-harmonique afin d'introduire des référents stylistiques propres au $m b u b e^{22}$, ajoutant ainsi quelques résonnances africaines qui lui sont familières parce que présentes au sein du patrimoine sonore afro-cubain: "Mais une fois que l'harmonisation de la chanson était faite, il fallait faire l'arrangement des cuivres, j’ai fait les voix, la trompette, le saxophone, le trombone. Aussi, l'arrangement des voix pour que ça sonne plus "Afrique" ». (Entrevue avec Méi-Ra St-Laurent, 18 février 2014)

\section{Nature du métissage}

En résumé, pour ce remixeur, le processus créatif part d'un langage mélodicoharmonique qu'il a développé en tant que musicien de formation jazz. Ce qui lui permet de relier les éléments stylistiques qu'il a choisis, c'est son arrangement à partir duquel il va agencer le folklore cubain avec celui du Québec, le tout en y ajoutant quelques éléments africains exprimant ainsi son goût pour la musique afro-cubaine qu'il a connue durant son enfance passée dans la région de Santiago de Cuba. Son espace créatif manifeste l'ensemble de ses idéaux: créer une musique jazz plus sophistiquée tout en intégrant l'essence de la musique populaire dansante, et ce, en conciliant sa situation d'immigré au Québec.

\section{TROISIÈME REMIXEUR: REM3}

\section{Présentation et contexte}

Comme il a été mentionné ci-haut, le troisième remixeur a bénéficié d'une formation spécialisée dans le cadre d'un Certificat en réalisation audionumérique offerte à la Faculté de musique de l'Université Laval, un programme où il a pu développer une expertise en mixage et en réalisation sonore. C'est dans l'objectif de pouvoir produire sa propre musique qu'il s'est inscrit à ce programme et nous ajoutons que pour lui, la musique possède une vocation particulière. En effet, il est animé par une démarche spirituelle progressiste très éloignée des stéréotypes et des préjugés largement diffusés par les médias depuis septembre 2001. Il s'intéresse à la musique populaire, mais d'un point de vue qui contraste avec les perceptions nord-américaines familières. Ceci mérite une explication supplémentaire. En avril 2009, nous avions l'occasion de préparer un documentaire pour le compte de TV5 monde sur l'émission Star Academy Arabia, alors diffusée au Liban. Notre recherche visait à mettre en contexte le concept de l'émission en tenant compte des réalités du monde arabe. Nous étions privilégiés de pouvoir travailler avec Georges Bassil, un des artisans de la prestigieuse production libanaise. Bref, nous avons découvert que pour les jeunes chanteuses et chanteurs du Star Academy Arabia, la participation à l'émission revêtait une tout autre signification que celle des jeunes nord-américains. En

22 Style choral que l'on retrouve en Afrique du Sud et qui a été largement diffusé, entre autres, grâce au film Le roi lion, dont la musique était d'Elton John, et au duo Simon and Garfunkel, dont l'album Graceland, paru en 1986, faisait référence à ce style sud-africain. 
effet, au Moyen-Orient, l'identification au concept de Star Academy possède une proposition symbolique progressiste, entre autres, à cause de la représentation de la femme "occidentalisée» employée dans un contexte où certains groupes islamistes jugent intolérable cette image "provocante» que projettent les jeunes dans l'ensemble des pays arabes par l'intermédiaire d'une émission diffusée à grande échelle. Ainsi, à l'instar de la musique raï, l'identification au modèle de musique populaire proposé par le Star Academy Arabia contient des germes beaucoup plus révolutionnaires qu'en Amérique par exemple, où l'industrie commerciale se base sur des standards visant la séduction d'une majorité d'auditeurs pour des raisons pécuniaires. Conséquemment, nous croyons qu'il faut tenir compte de cette situation pour mieux comprendre la démarche créatrice de Rem3.

\section{Esthétique, idéologie et identités}

Dans la conjoncture que nous venons de décrire, l'identification de Rem3 à la musique populaire revêt un sens particulier. Le choix d'une pièce de La Bolduc qui s'adresse aux ouvriers, et par extension au peuple, dans un langage dont la forme se veut plus accessible 23 , illustre bien une prise de position révélatrice. Pour nous, ce choix indique une prise de position consciemment engagée qui s'oppose aux comportements des promoteurs d'un l'islam élitiste et austère dont le mépris envers les classes sociales travailleuses n'est plus à démontrer. C'est donc avec une ferme insistance que Rem3 justifie son choix d'une musique joyeuse qui s'adresse avant tout au peuple:

La chanson est un hommage aux ouvriers. [...] Je n'écris pas bien le français, j’ai donc contacté un chanteur français avec lequel je pouvais travailler. Il m’a dit : «ça sonne très bien.» Je lui ai dit: «Tu dois parler de l'hommage aux ouvriers.» (Rencontre de groupe, 9 décembre 2009)

Je ne cherche pas un truc vraiment intellectuel difficile à écouter. (Entrevue avec Gérald Côté, 7 août 2013)

En réalité, je voulais que ça soit une chanson heureuse. Quand on l'écoute, je suis heureux, tu vois. [...] Et le thème est heureux. Ce sont ces gens-là qui ont bâti le Québec. (Entrevue avec Méi-Ra St-Laurent, 28 février 2014)

Comme il a été évoqué, la démarche de Rem3 s'inspire d'un prédicateur progressiste au sein du monde musulman, et ce, dans un objectif précis, celui de montrer une image différente des jeunes croyants musulmans:

Il s'appelle Amrokh Khalid. [...] Il est sorti du lot des prêcheurs classiques. J'avais 22 ans à cette époque. Il parlait au «je» et il inculquait la foi d'une manière très légère aux jeunes. [...] Il disait aux gens d'essayer de faire un truc bien pour l'islam dans leur domaine. Montrer que l'islam, ce sont des gens qui font de l'art, de la musique. (Entrevue avec Gérald Côté, 7 août 2013)

23 À cet égard, un peu plus loin dans cette entrevue, Rem3 insiste sur la simplicité langagière du prédicateur qu'il admire. 
C'est de manière parfaitement consciente qu'il dresse sa stratégie esthétique qui d'évidence n'est pas étrangère à sa vision progressiste de l'islam et dont l'objectif avoué est de «redorer l'image» des musulmans:

Le deuxième niveau est quand je fais des chansons qui sonnent bien et qui sont bien produites, c'est sûr que les gens vont se demander qui a produit cela. Ils vont voir que c'est «moi», un musulman. Ça rejoint un peu l'idée de redorer l'image et de faire une participation microscopique dans un truc qui est général. (Entrevue avec Gérald Côté, 7 août 2013)

Je dirai que c'est la première chose que nous a inculquée le prêcheur dont je vous ai parlé. C'est elle qui va sortir en vérité. C’est donner une image, un peu mieux qu'esthétique, et montrer que finalement les musulmans sont des gens qui créent, qui aiment la beauté. (Entrevue avec Gérald Côté, 7 août 2013)

C'est sans équivoque que cette prise de position, «un peu mieux qu'esthétique», comme il l'a formulée, revêt aussi des valeurs religieuses et politiques pour lui :

Montrer que les musulmans ont du goût. Ils aiment les belles choses. Ils aiment créer. Il y a aussi un autre truc qui est vraiment plus profond que ça. C'est montrer la gloire de Dieu. Je dirais que c'est un deuxième niveau qui n'est pas encore révélé à cent pour cent. J’aimerais avoir ce genre d'approche dans mon travail, mais ce n'est pas ce que je ressens maintenant. C'est comme s'il y avait trois niveaux qui étaient tous plus profonds les uns que les autres. Le deuxième niveau est plus social, plus politique. Montrer que les musulmans peuvent faire de beaux trucs, que ce n'est pas toujours ce qu'on pense d'eux. Quand tu crains un truc et tu te dis: "Qui l'a fait? C'est un musulman». C'est comme participer un peu à redorer l'image. (Entrevue avec Gérald Côté, 7 août 2013)

C'est à l'aide du langage harmonique populaire, âprement proscrit par l'ascèse islamique, que le résultat escompté se veut une expression protestataire à l'égard d'un monde qu'il faut changer. Bien plus, nous croyons que la modernisation de la musique de Madame Bolduc constitue une véritable métaphore de son idéal quant à l'avenir de l'islam:

Je dirais que c'est un remix de l'ancienne [version] [...]. Je me suis dit, c'est comme si La Bolduc me disait: «J'aimerais moderniser cette chanson et la chanter, pour voir comment ça va être». Les accords, je les ai changés un tout petit peu [...], sont une chaîne d'accords pop modernes. (Entrevue avec Méi-Ra St-Laurent, 28 janvier 2014)

À son amalgame des genres susceptibles de réunir l'ancien et le nouveau, Rem3 réfère consciemment à plusieurs styles pouvant soutenir sa vision de la modernité, et ce, tout en introduisant une fonction de divertissement qui inclut des rythmiques permettant la danse:

Toujours dans la même formule, je regarde les accords principaux de l'ancienne chanson, la façon dont elle a été créée et un petit changement d'accords seulement pour la moderniser. Il te donne la base. Donc, sur ça 
j'ai ajouté un petit morceau d'accordéon. Tout ça dans un style de «housedance». [...] Vraiment, c'est [...] de la pop, de l'électro-pop, mais ce n'est pas vraiment une musique électronique, parce que le rythme n'est pas vraiment celui de cette musique. Je crois que c'est juste 100 BPM dans le tempo. Ce n'est pas du 120 ou 125. Le tempo n'est pas très rapide, mais c'est vraiment de l'électro-pop acoustique avec une guitare acoustique [...]. J'essaie toujours d'ajouter des éléments qui optimisent le groove. Ça doit vibrer. (Entrevue avec Gérald Côté, 7 août 2013)

\section{Nature du métissage}

Il nous apparaît donc impossible de dissocier la musique de Rem3 de ses idées prospectives, c'est-à-dire de sa vision modernisée de l'islam et de sa critique des politiques islamisées relatives aux réalités contemporaines depuis septembre 2001. Assurément, le jeu de combinaison et de transformation des différents éléments stylistiques possède une fonction particulière dans le discours sonore du remixeur, c'est-à-dire que le renouvellement de l'ancien, tel que conçu par Rem3, évoque la modernisation de l'islam au sein du monde contemporain. Cela a pu se faire grâce à des référents stylistiques provenant de la musique populaire internationale et dont l'espace sémantique est envisagé comme une forme d'opposition de nature symbolique à la vision des fondamentalistes. Ainsi, dans le contexte actuel de la montée de l'intégrisme islamique sur le plan international, il s'agit, pour Rem3, d'un désir bien assumé de manifester autrement ses propres croyances tout en préservant sa foi initiale en Allah. Assurément, ce remix constitue une mise en scène qui est en parfaite conformité avec la vision du monde d'un jeune musulman progressiste établi au Québec depuis plus d'une dizaine d'années.

\section{QUATRIÈME REMIXEUR : REM4}

\section{Présentation et contexte}

Rem 4 a fait des études universitaires en philosophie, s'intéressant entre autres à l'esthétique, à la phénoménologie et à la philosophie analytique. Après avoir terminé sa maîtrise sur mesure en réalisation audionumérique à l'Université Laval, à Québec, il a entrepris un doctorat en recherche-création. Il souhaite approfondir ses recherches sur une technique qu'il a développée au LARC et dont l'objectif est de faire en sorte que le matriçage puisse se faire au cours de toutes les étapes du processus de production (design des sons, prises de son, mixage). Nous croyons que cette idée est en lien direct avec sa conception de la création. En effet, grâce à cette façon de concevoir le matriçage, il s'assure de conserver l'intégrité des textures sonores initiales, souvent compromises par le traitement dynamique et spectral lors de cette dernière étape de la production. Or nous croyons que cela n'est pas étranger à une certaine conception de l'œuvre faisant appel à une réflexion esthétique bien connue sur l'art et qui a ses assises dès le début du siècle (Benjamin 1935) ${ }^{24}$. Et comme le sous-entend

24 Les œuvres de la modernité intègrent souvent un discours sur elles-mêmes et sur l'art. Les mouvements de la modernité recherchent, tant sur le fond que la forme, un art épuré se rapprochant de ses fondements. (Trépanier et Vaillancourt 2000, p. 18) 
sa démarche, qui était déjà manifeste dans son projet de maîtrise, pour Rem4, l'esthétique individuelle n'est pas négociable, les idées initiales du compositeur doivent être préservées des traitements déformants que permet la technologie. À ce propos, lors d'une conférence donnée à Oslo, il expose cette idée où le mixage et le matriçage font partie intégrante de la démarche artistique: «Nous considérons le processus phonographique, y compris mixage et le mastering, comme un art créatif, plutôt que de le considérer simplement comme un processus "reproductif" qui est plus ou moins authentique et performantiel.» (Oslo ${ }^{25}, 4$ décembre 2014)

Ainsi, pour ce dernier, les équipements en studio qui modulent le processus créatif risquent de faire subir des mutations indésirables aux idées initiales du créateur, ce qui relève pour nous d'un point de vue personnel qui consent une valeur intrinsèque à l'inspiration, à l'intuition ainsi qu'à l'art qui en découle. En entrevue, il admet ne pas vouloir penser à ce qu'il va faire afin de s'éloigner de toute forme d'idées reçues, et ce, même s'il crée à partir de référents stylistiques existants qu'il a choisis intuitivement. Il avoue aussi qu'il ne veut pas emprisonner son imagination ni la compromettre par un usage excessif de la technologie.

\section{Esthétique, idéologie et identité}

La démarche artistique de Rem4 se manifeste premièrement dans le choix des éléments qu'il aura à remixer. Se fiant à son sens artistique, s'appuyant sur ses intuitions, c'est avec une certaine difficulté qu'il cherche son matériel de base pour sa composition, mais sans y ajouter un jugement de valeur, même s'il ne se reconnaît pas dans le répertoire proposé:

Je n’aime pas tant la musique québécoise. Je n’ai aucune culture là-dedans. Puis, je n'ai pas assez de connaissances pour au moins l'apprécier d'un point de vue analytique. Même d'un point de vue de sa sonorité, du genre, je n'aime pas ça. Je ne comprends pas pourquoi. (Entrevue avec Méi-Ra St-Laurent, 8 avril 2014)

Dans le choix des éléments qu'il va prendre pour son amalgame sonore, il cherche consciemment à éviter la facilité et les pièges du "déjà-vu», des critères déterminant qui le mèneront à des référents musicaux plus actuels. Aussi, il est préoccupé par l'idée d'ajouter à ses préférences esthétiques, un caractère qu'il considère avec humour de "funk dangereux» :

C’est peut-être parce que j'ai «stické» pas mal sur le style électronique. Je me disais que si j'en faisais un hip-hop instrumental ça serait vraiment facile. Ça ne me prendrait vraiment pas beaucoup de temps et ça irait mieux déjà, mais j'aimerais ça en faire de quoi plus funk électronique même un peu dansant et dangereux. (Rencontre de groupe, 15 octobre 2012)

25 "The 9th Art of Record Production Conference: Record Production in the Internet Age», Association for the Study of Record Production (ASARP), 4-6 décembre 2014. 
Dans une attitude autocritique, il nous confie que ses choix stylistiques pour le projet «Remixer la chanson québécoise» sont à l'opposé de la musique «trash ${ }^{26} »$ du point de vue de la texture et de la dynamique, un genre qu'il utilisait très récemment pour ses compositions:

J'ai choisi des ambiances plus douces, plus lentes parce que j'étais saturé dans ma période plus «trash» où je me suis bien amusé, mais maintenant je trouve ça un peu stupide. (Entrevue avec Gérald Côté, 4 octobre 2014)

J'ai essayé un peu de changer de "mood» dans mes affaires en général. Avant je tripais sur les trucs un peu "trash», genre "destroy». Je me suis rendu compte que [...] maintenant je trouve ça idiot. (Rencontre de groupe, 15 octobre 2012)

On retrouve d'ailleurs ce passage du «trash» au doux dans l'exercice même de sa composition pour le projet:

Pour ce qui est de la composition, j'avais commencé une pièce de dubstep très violente, mais j'ai dû me rendre à l'évidence que le contraste entre les styles n'était vraiment pas de mon goût, et malgré plusieurs essais, j'ai décidé de changer de style. Ces temps-ci, je fais un peu de musique électronique plus ronde/soft, alors je crois que je vais suivre cette direction également pour les remixes, afin d'obtenir une fusion plus harmonieuse entre les deux styles. (Page des remixeurs, 30 novembre 2011)

Ainsi, les sonorités recherchées pour son remix s'orientent résolument vers des ambiances plus soyeuses, soutenues par un «down tempo » et aux couleurs jazzy, bluesy, auxquelles il ajoute des éléments hip-hop et trip hop:

Mes idées de remix étaient inspirées par mes écoutes actuelles (Mushroom Jazz par Mark Farina dans hip-hop UK 1990, et les compilations Jazz Cafe). Mon remix est aussi causé par des besoins fonctionnels: le hiphop s'agence bien avec des échantillons très variés, tout en restant de bon goût. (Page des remixeurs, 30 novembre 2011)

C’est vraiment [...] je dirais hip-hop, trip hop, euh [...]. C’est pas mal ça je pense. Pis jazz. Mais ça rentre pas mal tout dans le hip-hop. C'est du hip-hop, mais genre échantillonné avec du jazz. (Entrevue avec Méi-Ra St-Laurent, 8 février 2014)

Je tripe vraiment sur les trucs UK/9o qui sonnent même à la limite un peu $\mathrm{R} \& \mathrm{~B}$, mais avec des «simples» vraiment jazz et ça ressemble drôlement à ce que j'ai apporté. (Rencontre de groupe, 15 octobre 2012)

26 La référence à un style "poubelle» possède une connotation hypermoderne qui trahit une certaine vision du monde actuel. Le concept se retrouve d'ailleurs dans plusieurs formes artistiques, dont la littérature et l'art visuel. (Voir Fernandez Porta 2008, p.11). 


\section{Nature du métissage}

Dans l'ensemble, les entrevues indiquent une approche singulière où l'intégrité artistique se manifeste dans la manière de faire les choses plus que par les référents sonores que Rem4 relativise lors des entrevues. Ces référents esthétiques s'opposent entre eux de manière contrastée, admet-il d'ailleurs. À notre avis, cela rejoint les réalités postmodernes et hypermodernes décrites dans notre exposé théorique. Or ses choix esthétiques se font selon une démarche identitaire qui s'affirme, entre autres, par le contournement des pièges de la standardisation et de ses formats prédigérés, ipso facto, par la réappropriation de modèles stylistiques qu'il considère nouveaux et intéressants. Ce souci d'intégrité artistique est manifeste à toutes les étapes de son processus créatif, s'appuyant ainsi sur un modus operandi qu'il avait développé lors de ses recherches universitaires.

\section{RETOUR RÉFLEXIF}

Bien que les quatre remixeurs possèdent des démarches distinctives en regard de leur contexte et de leur conditionnement socioéducatif, tous considèrent leur propre travail créatif comme un jeu d'assemblage d'éléments sonores ou stylistiques, ce que d'ailleurs nous considérons comme étant la structure superficielle relative à toute forme de création musicale ou autre, comme nous l'avons déjà évoqué. Ramassés sur le chemin d'histoires singulières, les fragments de musiques réconciliés par les remixeurs ne sont pas neutres. Ils ne le sont pas, ni séparément ni une fois assemblés, au profit de la nouvelle structure symbolique et significative obtenue par leur remix. Bien que l'exercice en luimême exige un travail de fusion d'éléments diversifiés chez des individus aux parcours très différents, ce qui est remarquable est que nous avons pu observer une configuration similaire au sein du processus créatif de tous les remixeurs. Premièrement, les choix des pièces à remixer se sont faits en parfaite conformité avec la perception qu'ils ont des réalités postmodernes et hypermodernes ainsi que de leur rôle au sein de cette réalité. En effet, quelles que soient leurs expériences singulières, les éléments esthétiques qu'ils ont choisi de réunir dans leur composition l'ont été en regard de leur histoire personnelle (de la perception qu'ils ont d'eux-mêmes dans le monde), ce qui a rendu ces éléments significatifs, d'ailleurs, dans leur processus de construction identitaire. La motivation sous-jacente aux choix de ces éléments significatifs pour eux peut être de nature philosophique, idéologique, politique ou encore psychique, mais ce sur quoi nous insistons, c'est que nous croyons que c'est à ce niveau de profondeur que l'ordre semble émaner du chaos afin d'en faire émerger un sens en particulier. Cette dimension du processus créatif se fait évidemment à des degrés de conscience différents, dans un spectre pouvant osciller entre l'intuition et le discours délibéré. Bien qu'invisibles, ces éléments qui motivent la créativité sont bien ancrés dans l'humanité sociétale de chacun des remixeurs, que ce soit pour servir une critique sociale (Remi), pour partager une forme artistique où le plaisir hédoniste de danser se joint à un jazz plus savant (Rem2), pour manifester un idéal où la croyance religieuse se réconcilie avec le monde 
contemporain (Rem3), ou encore pour élaborer une construction sonore sans compromis dans une démarche esthétique personnelle en situation d'expérimentation technique (Rem4). Pour tous ces remixeurs, ce qui réconcilie les différents éléments ne vient pas de l'extérieur de l'individu, mais de l'intérieur, c'est-à-dire de leur identité profonde. Il s'agit bien pour chacun d'eux d'une vision particulière du monde qui structure les éléments sonores éclectiques en une nouvelle forme.

Or nous avons remarqué un autre aspect présentant un élément commun dans la démarche de ces quatre remixeurs et qui relève aussi d'un mécanisme d'individuation. Il s'agit du phénomène de construction identitaire qui se crée "par opposition», mettant en relief «une différence» en rapport avec un pôle existant dans le monde social. Dans cette dynamique, l'esthétique individuelle s'affirme par le biais de jeux d'opposition significatifs, susceptibles de marquer une position sociale ou une perception distinctive du monde. Par exemple, pour Rem1, la démarche esthétique épouse les mouvements sociocritiques des années 1950 à 1970 qui s'opposent aux abus liés aux pouvoirs religieux et capitalistes par des manifestations artistiques symboliques. Pour Rem2, il s'agit, par la nature même de ses principaux choix stylistiques, soit de la musique populaire et du jazz, de l'expression de son opposition au pouvoir politique cubain qui rejette ces styles pour des raisons idéologiques. Assurément, son résultat esthétique constitue une forme d'opposition à l'impérialisme culturel étatiste. Rem3 choisit lui aussi une formule esthétique antagoniste quant aux exigences hiératiques des intégristes islamiques, c'est-à-dire une musique qui s'adresse à tous et où le plaisir et la beauté forment un couple «moderne» qui s'oppose à une doctrine ancienne où le fondamentalisme et le politique se confondent. Finalement, Rem 4 fait ses choix stylistiques dans une dynamique où ces derniers se présentent face à lui-même, passant de styles «trash" à des ambiances plus soyeuses au rythme plus lent. Conséquemment, nous croyons que les jeux de polarités antagoniques sont d'excellents révélateurs du phénomène de construction identitaire.

En conclusion, tous les remixes sont des assemblages de référents culturels qui ont été restructurés par les musiciens au profit d'un discours relevant d'une vision singulière du monde dans un contexte délimité. Chaque remix est né d'une intention qui se manifeste par l'intermédiaire d'une reconfiguration d'éléments musicaux possédant des qualités discursives contextuelles. C'est d'ailleurs à partir de cette intention qu'émergent non seulement la motivation, mais aussi un sens quelconque, un sens qui s'articule autour d'une identité, un sens qui existe dans la profondeur du moi-individu-social et qui n'est jamais complètement atteignable par la surface esthétique d'un objet sonore composite.

\section{RÉFÉRENCES}

Aubert, Nicole. décembre 2004. "L'individu hypermoderne : vers une mutation anthropologique?», Sciences humaines, $\mathrm{n}^{\circ}$ 154: 36-41. Repris dans: Xavier Molénat (coord.) mai 2006. L'individu contemporain, Regards sociologiques. Éditions Sciences Humaines. 
Bhabha, Homi. 1989. «Hybridité, identité et culture contemporaine». Exposition «Magiciens de la terre», 24-27. Paris: Musée national d'art moderne, Centre George-Pompidou.

Blacking, John.1980. Le sens musical. Paris: Les Éditions de Minuit. Borduas, Paul-Émile,1948. Refus global. Montréal: Librairie Henri Tranquille. Boulez, Pierre.199o. «Mémoire et création», Cours de 1990, 7 -65. Collège de France.

—.1989. "Mémoire et création», Cours de 1989, 2-53.Collège de France.

Bourdieu, Pierre.1984. Questions de sociologie. Paris: Les Éditions de Minuit.

Bourricaud, François. 1969. Le bricolage idéologique. (Essai sur les intellectuels et les passions démocratiques). Paris: Presse Universitaire de France.

Burke, Peter J. et Jan E. Stets. 2009. Identity Theory. New-York : Oxford University Press.

Calvet, Louis-Jean. avril 1985. «Métissage et colonisation». Vibrations, no 1: 2227. Toulouse: Privat.

Constantin, Denis. 2001. "Le métissage en musique: un mouvement perpétuel ». Cahier d'ethnomusicologie, no 13:3-33.

Côté, Gérald. 2011. Le jazz vu de l'intérieur. Pour une anthropologie des musiques afro-américaine. (Réédition augmentée). Québec: Varia.

—. 2003. "Le métissage vu comme lieu du discours idéologique». Construire le savoir musical : enjeux épistémologiques, esthétiques et sociaux, sous la dir. de Monique Desroches et Ghyslaine Guertin. Paris: L'Harmattan, collection Logiques Sociales.

—. décembre 2001. «Métissage à la Québécoise: Histoire, crises et composites». Les Cahiers de la Société québécoise de recherche en musique $4, \mathrm{n}^{0} 2$.

—.1998. Processus de création et musique populaire : un exemple de métissage à la québécoise. Paris: L'Harmattan.

Csikszentmihalyi, Mihaly. 1988. "Society, Culture, and Person: A Systems View of Creativity». The

Nature of Creativity, sous la dir. de Robert J.Sternberg, 325-339. New York: Cambridge University Press

Cubana Bop Partners.1988. «Dizzy Gillespie : à nous deux La Havane». Cubana Bop Partners, Réalisation: Nim Polanetska, durée 84 minutes.

Dumont, Fernand.1996.Genèse de la société québécoise. Montréal: Boréal.

Edmond, Marc. 2005. Psychologie de l'identité. Soi et le groupe. Dunod: Presse universitaire de France.

Ehrenreich, John et Barbara Ehrenreich. 1979. Between Labor and Capital, sous la dir. de Pat Walker. Boston: South End Press.

Frith, Simon. 2004. «Popular Music and Identity». Psycology Press, Vol. 4 (Critical Concepts in Media and Cultural Studies). Oxford : Routledge.

Hennion, Antoine. Avril 1985. «Dossier : Métissage et musiques métissées», Vibrations, $\mathrm{n}^{\mathrm{0}}$ 1: 11-243. Toulouse: Privat.

Fernandez Porta, Eloy. 2011. Homo Sampler. Culture et consommation à l'ère afterpop. Paris: Édition inculte.

Lipovetsky, Gilles.2004. Les temps hypermodernes. Paris: Grasset. 
Lochhead, Judy. 4 octobre 2010 "Cycles de conférences Grands Témoins sur le thème de l'hypermodernité». Écully: Institut Paul Bocuse.

Lysloff, Gay.2003. Music and techno culture. Middletown: Wesleyan university Press.

Longhi, Julien .2008. Objets discursifs et doxa - essai de sémantique discursive. Paris: L'Harmattan.

Nomani, Ashra. May 2007. "The Most Influential People in The World, Heroes and Pionneers". Time Magazines, The TIME 100.

Paillé, Pierre et Alex Mucchielli. 2008. L'analyse qualitative en sciences humaines et sociales ( $2^{\text {ème }}$ éd.). Paris: Armand Colin.

Simonton, Dean K. 1988. "Creativity, Leadership, and Chance», The Nature of Creativity, sous la dir. de Robert J.Sternberg, 386 à 428. New York : Cambridge University Press.

Sternberg, Robert J. 1988. "The Nature of Creativity». Contemporary Psychological Perspectives, New York: Cambridge University Press.

Stryker, Sheldon.1980. Symbolic interactionism: A social structural version. Cadwell, NJ: Blackburn Press.

Timothy D. Taylor.2002. "Music and Musical Practice in Postmodernity». Postmodern Music/Postmodern Thought, sous la dir. de Judith Irene Lochhead et Joseph Henry Auner, 93-118. New York: Routledge

Trépanier, Michel et Claude Vaillancourt. 200o. La modernité et le surréalisme. Laval: Éditions études vivantes.

\section{RÉSUMÉ}

Cet article présente une analyse contextuelle du travail créatif de quatre musiciens qui avaient comme mandat de remixer des chansons québécoises enregistrées avant les années 1960. Par le biais d'une enquête dont l'approche s'inspire de méthodes ethnographiques, l'auteur tente d'entrer dans la tête et le cœur des musiciens en vue d'identifier ce qui motive leur créativité et ce qui mène ces derniers à s'incliner vers certains modèles esthétiques plutôt que d'autres. Dans un espace social qualifié de postmoderne ou encore d'hypermoderne selon les sociologues, les motivations nous apparaissent diversifiées, fragmentées, fluctuantes et très contrastées d'un musicien à l'autre, et ce, à l'image d'une époque, d'une génération ou d'une réalité anthropologique urbaine et contemporaine. Lauteur conçoit la musique comme un phénomène multifactoriel où le social et le musical sont interdépendants, en mouvements ainsi qu'en constantes mutations.

\section{ABSTRACT}

This article presents a contextual analysis of the work of four musicians, who have been asked to remix Quebec songs recorded before the 196os. Through an examination drawing on ethnographic methods, the author tries to understand what makes musicians tick, by attempting to identify what motivates their creativity and what leads them to choose certain aesthetic styles over others. In a social environment qualified by sociologists as postmodern or ultramodern, the motivations appear diverse, 
fragmented, fluctuating, and highly contrasted from one musician to another, reflecting our anthropologically contemporary and urban times, generations, and realities. The author conceives music as a multifactorial phenomenon in which music and society are interrelated, and in constant movement and transformation.

\section{BIOGRAPHIE}

Gérald Côté a obtenu son doctorat en ethnomusicologie en 1997. Il publie plusieurs essais dont Les 101 blues du Québec et Processus de création et musique populaire, ainsi que de nombreux articles et conférences données en Roumanie, au Brésil, au Mexique et en Afrique. Il est le codirecteur et cofondateur du Centre de recherche ethnomusicologique Acte Sept (CREAS) depuis 1995, un centre d'étude situé à Bamako sur la pratique traditionnelle des musiques maliennes, ce qui l'a amené à passer plusieurs séjours sur la côte ouest-africaine. En 2011, il a travaillé à la conception d'une exposition internationale au Musée de la civilisation de Québec sur le thème de l'influence musicale de l'Afrique sur la musique des continents américains. Le succès de son dernier livre Jazz vu de l'intérieur-pour une anthropologie des musiques afro-américaines, lui a assuré une réédition en octobre 2011 et a fait l'objet de conférences au Berklee Colledge of Music de Boston. En tant que professeur invité à l'Université Laval de Québec, il poursuit toujours ses recherches sur ce qui motive la création des différentes musiques au sein d'une approche multifactorielle et holistique. 